\title{
Robust interactive image segmentation via graph-based manifold ranking
}

\author{
Hong $\mathbf{L i}^{1}(\bowtie)$, Wen $\mathbf{W u}{ }^{1}$, and Enhua $\mathbf{W u}^{1,2}$ \\ (c) The Author(s) 2015. This article is published with open access at Springerlink.com
}

\begin{abstract}
Interactive image segmentation aims at classifying the image pixels into foreground and background classes given some foreground and background markers. In this paper, we propose a novel framework for interactive image segmentation that builds upon graph-based manifold ranking model, a graph-based semi-supervised learning technique which can learn very smooth functions with respect to the intrinsic structure revealed by the input data. The final segmentation results are improved by overcoming two core problems of graph construction in traditional models: graph structure and graph edge weights. The user provided scribbles are treated as the must-link and must-not-link constraints. Then we model the graph as an approximatively k-regular sparse graph by integrating these constraints and our extended neighboring spatial relationships into graph structure modeling. The content and labels driven locally adaptive kernel parameter is proposed to tackle the insufficiency of previous models which usually employ a unified kernel parameter. After the graph construction, a novel three-stage strategy is proposed to get the final segmentation results. Due to the sparsity and extended neighboring relationships of our constructed graph and usage of superpixels, our model can provide nearly real-time, user scribble insensitive segmentations which are two core demands in interactive image segmentation. Last but not least, our framework is very easy to be extended to multi-label segmentation, and for some less complicated scenarios, it can
\end{abstract}

1 Department of Computer and Information Science, University of Macau, Macau 999078, China. Email: H. Li, valhongli@gmail.com (凶); W. Wu, wenwu@umac.mo.

2 Chinese Academy of Sciences, Beijing 100000, China. Email: ehwu@umac.mo.

Manuscript received: 2015-08-11; accepted: 2015-09-15 even get the segmented object through single line interaction. Experimental results and comparisons with other state-of-the-art methods demonstrate that our framework can efficiently and accurately extract foreground objects from background.

Keywords interactive image segmentation; graph structure; graph edge weights; manifold ranking; relevance inference

\section{Introduction}

Image segmentation, which is described as extracting meaningful partitions from an image, is one of the most fundamental, well-studied but challenging problems in image processing and computer vision. In general, image segmentation models can be divided into two groups: automatic and interactive segmentations. There are many models in each group and $\mathrm{Hu}$ et al. [1] presented a very comprehensive review. For automatic image segmentation approaches, which are known as unsupervised models, they automatically classify the image pixels into coherent regions without any prior knowledge, such as mean shift $[2,3]$, level sets [4$6]$, and graph based methods [7, 8]. Although automatic image segmentation models have gained much success and been applied to many other algorithms, such as scene parsing [9], they are still far away from satisfaction; especially they have the problem of separating the whole object into different parts. On the other hand, interactive segmentation methods can accurately extract the whole object from the input image based on the user provided prior knowledge about the object and background. So in this paper, we only focus on interactive image 
segmentation models, in the sense that the users provide a partial labeling of the image.

Recently, interactive image segmentation methods have gained an extensive popularity since the methods give users the ability to affect the segmentation results as necessary for given applications. However, image segmentation is not easy because of many difficulties, such as noise pollution, illumination variation, and background clutter, and so on. In the meanwhile, the segmentation results should also not be sensitive to the seed location and quantity in order to reduce the user effort. To confront all these difficulties, many approaches have been proposed in the literature with impressive results. Popular approaches include graph-cut based methods [1014], edge based methods [15-17], random walk based methods [18-20], and region based methods [21-23].

Almost all of these existing interactive segmentation systems provide users with an iterative procedure to add or remove scribbles to temporary results until they get the final satisfactory segmentation result. However, they can only get high-precision segmentation results at the cost of high computational complexity or many carefully placed seeds. Obviously, these two disadvantages make their models impractical because the users usually require the system to respond quickly and update the corresponding result immediately for further refinement. In another word, the system should have an acceptable computational complexity, even real-time performance, of interactive segmentation algorithms.

In order to overcome these shortcomings, we propose a robust interactive image segmentation system that builds upon graph-based semisupervised learning theory and superpixels. Figure 1 illustrates the framework of our proposed model. The input image is firstly over-segmented into small homogeneous regions and the user provided scribbles are integrated with superpixels. Then we model the approximately k-regular sparse graph and form the affinity graph matrix using proposed labels driven and locally adaptive kernel parameter. The final segmentation is generated by a three-stage strategy.

Contributions. The key contributions of this work are:

1. A novel framework that combines the graph-

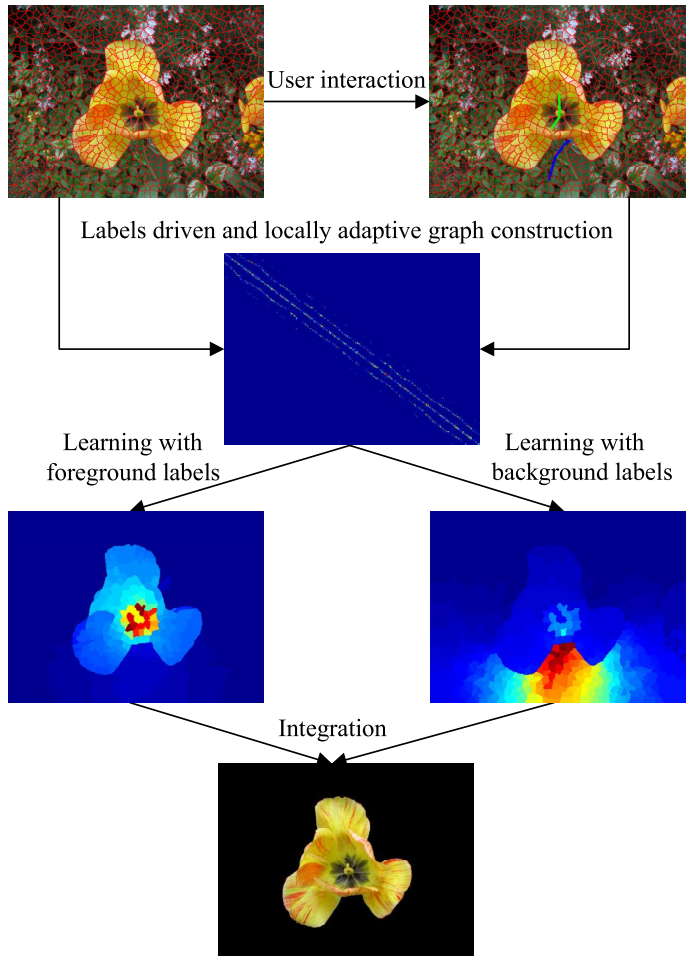

Fig. 1 The framework of our proposed interactive image segmentation.

based semi-supervised learning theory with region based models to efficiently segment out the desired object(s) accurately. It can be easily extended to multi-label segmentation and single-line cutout problems.

2. A novel graph construction strategy which models the graph as an approximately k-regular sparse graph by integrating spatial relationships and user provided scribbles.

3. A new graph edge weights computing strategy which forms the weights using a locally adaptive kernel width parameter.

The rest of the paper is organized as follows. First, related works are summarized in Section 2. In Section 3, we first introduce the basic concept of graph-based manifold ranking in Section 3.1 and then give the details of our three-stage interactive image segmentation framework in Section 3.2. Experimental results and analysis are given in Section 4. Finally, conclusions and future work are given in Section 5 .

\section{Related work}

Related works fall into two categories: segmentation 
based on graph theory and region operations. We address each of them in turn.

Graph based segmentation models can be roughly divided into two subgroups: graph-cut based models and random walk based models. Boykov and Jolly [10] propose the first interactive graphcut model. The user provided foreground and background seeds are treated as source and sink nodes in graph respectively and the segmentation is performed by the min-cut/max-flow algorithm. It has been very popular because of its strong mathematical foundation provided by the maximum a posterior-Markov random field (MAP-MRF) framework [24]. Rother et al. [11] propose an iterated graph-cut algorithm named GrabCut. It uses a Gaussian mixture model (GMM) to model the pixel colors' distribution and alternates between object estimation and GMM parameter estimation iteratively. GrabCut needs less user interaction by only requiring a rectangle or lasso around an object (not detailed foreground and background seeds). Li et al. [12] also propose an improved (both in speed and accuracy) interactive graph-cut algorithm named Lazy Snapping. They adopt superpixels to construct the graph to reduce the computational cost. It also supports boundary editing to achieve pixel-level accuracy. All these graph-cut based methods sometimes have the problem of shortcutting and it is usually caused by a lower cost along a shorter cut than that of a real boundary. To overcome this problem, Price et al. [13] propose a geodesic graph-cut method which takes geodesic distance (instead of Euclidean distance) into account. It outperforms previous graph-cut based methods when user provided information separates the background and foreground feature distributions effectively.

Random walk based methods classify an unlabeled pixel via resolving a question: if a random walker starts from one location, what is the most probable seed destination? Grady [18] regards the image segmentation as random walk on a graph and demonstrates that the method is more robust to noise, weak boundary detection, and ambiguous region segmentation. However, it is very sensitive to the seeded points. Kim et al. [19] propose a generative image segmentation algorithm by utilizing random walk with restart (RWR) which gives the walker two choices: randomly move to one of its neighbors with probability $c$ or jump back to its initial seed point and restart with probability $1-$ c. RWR algorithm can segment images with weak boundaries and textures more effectively, but its computational cost is very high because it demands large matrix inversion.

Region based methods can be categorized into two subgroups: region growing, region splitting and merging. Adams and Bischof [21] propose a fast and easily implemented method based on region growing. It iteratively adds pixels in subregions near the foreground or background subregions to the active set and updates the seeds until all pixels in the image are assigned to a label. It generates unsatisfactory results when foreground and background have close color distribution. Both maximal similarity-based region merging (MSRM) [22] and mating attributed relational graph (MARG) [23] begin with superpixels (the input image is divided into small homogenous regions). MSRM iteratively merges a region into a neighboring region which has the most similar color histogram and updates the histogram of newly merged region until there is no region to be merged. It has high overall computational complexity because it needs computing the histogram similarity in each iteration. MARG constructs two graphs: the input graph, which represents the input superpixels image; and the model graph, which is constructed by the labeled superpixels. Then the region merging is performed by matching these two graphs. This method needs many labeled pixels which is not impractical.

\section{Robust interactive image segmentation via graph-based manifold ranking}

In this section, we first briefly introduce the graphbased manifold ranking in Section 3.1, then present the details of our proposed three-stage interactive image segmentation framework in Section 3.2.

\subsection{Graph-based manifold ranking}

Graph-based semi-supervised models usually consist of two main parts: graph modeling and information inference. Given a set of $n$ data points $X=$ 
$\left\{x_{1}, x_{2}, \cdots, x_{q}, \cdots, x_{n}\right\}$, with each data $x_{i} \in R^{m}$, the first $q$ points $\left\{x_{1}, x_{2}, \cdots, x_{q}\right\}$ are labeled as the queries and the rest points $\left\{x_{q+1}, \cdots, x_{n}\right\}$ are unlabeled. The ranking algorithm aims to rank the remaining points according to their relevances to the labeled queries. Let $f: X \rightarrow R^{n}$ denotes a ranking function which assigns to each data point $x_{i}$ a ranking value $f_{i}$. We can treat $\boldsymbol{f}$ as a vector $\boldsymbol{f}=$ $\left[f_{1}, f_{2}, \cdots, f_{n}\right]^{\mathrm{T}}$. We can also define an indication vector $\boldsymbol{y}=\left[y_{1}, y_{2}, \cdots, y_{n}\right]^{\mathrm{T}}$, in which $y_{i}=1$ if $x_{i}$ is a query, and $y_{i}=0$ otherwise.

Next, we define a graph $G=(V, E)$ on these data points, where the nodes $V$ are dataset $X$ and the edges $E$ are weighted by an affinity matrix $\boldsymbol{W}=\left[w_{i j}\right]_{n \times n} . \quad \boldsymbol{W}$ is often obtained by applying the Gaussian kernel to a distance matrix:

$$
w_{i j}=\mathrm{e}^{-\frac{d^{2}(i, j)}{\sigma^{2}}}
$$

where $d(i, j)$ denotes the distance between $x_{i}$ and $x_{j}$ and usually is computed via Euclidean distance between colors, $\sigma$ decides the kernel size. The degree matrix is denoted as $\boldsymbol{D}=\operatorname{diag}\left\{d_{1}, d_{2}, \cdots, d_{n}\right\}$, where $d_{i}=\sum_{j=1}^{n} w_{i j}$.

According to Zhou et al. [25, 26], cost function associated with the ranking function $f$ is defined to be

$$
\begin{aligned}
Q(\boldsymbol{f})= & \frac{1}{2}\left(\sum_{i, j=1}^{n} w_{i j}\left\|\frac{1}{\sqrt{d_{i i}}} f_{i}-\frac{1}{\sqrt{d_{j j}}} f_{j}\right\|^{2}\right. \\
& \left.+\mu \sum_{i=1}^{n}\left\|f_{i}-y_{i}\right\|^{2}\right)
\end{aligned}
$$

where the regularization parameter $\mu>0$ controls the balance of the first term (smoothness constraint) and the second term (fitting constraint, containing labeled as well as unlabeled data.). The first term means that nearby points should have similar scores. Then the optimal ranking $f^{*}$ of queries is computed by solving the following optimization problem:

$$
f^{*}=\arg \min _{f} Q(\boldsymbol{f})
$$

The solution of Eq. (3) can be denoted as

$$
f^{*}=(\boldsymbol{I}-\alpha \boldsymbol{S})^{-1} \boldsymbol{y}
$$

where $\boldsymbol{I}$ is an identity matrix, and $\boldsymbol{S}=\boldsymbol{D}^{-\frac{1}{2}} \boldsymbol{W} \boldsymbol{D}^{-\frac{1}{2}}$ is the normalized Laplacian matrix, $\alpha=1 /(1+\mu)$. The detailed derivation can be found in Refs. $[25,26]$.

\subsection{Segmentation via graph-based manifold ranking}

Above mentioned graph-based semi-supervised learning algorithm indicates that our interactive image segmentation framework should consist of two main parts: graph construction and information inference. In Section 3.2.1, we present our labels driven and locally adaptive graph construction. And in Section 3.2.2, we present our three-stage interactive image segmentation procedure.

\subsubsection{Labels driven and locally adaptive graph construction}

To better exploit the intrinsic relationship between data points, there are two aspects should be carefully treated in graph construction: graph structure and edge weights. We over-segment input image into small homogeneous regions using work [27] instead of popular simple linear iterative clustering (SLIC) model [28] because superpixels generated by work [27] have better boundary fitness than that of work [28]. Then we regard each superpixel as a node in the graph $G$.

For graph structure, we take the local smoothness cue (i.e., local neighboring superpixels are more likely to belong to the same object) into account and follow three rules. Firstly, each node is not only connected with its direct adjacent neighboring nodes, but also is connected with those nodes sharing common boundaries with its neighboring nodes. Secondly, the nodes labeled as foreground should be connected and the nodes labeled as background should also be connected. Thirdly, the labeled foreground and background nodes should not be connected. First rule models the graph as a k-regular structure by extended neighboring relationships and makes sure the graph structure being sparse. The rest two rules integrate the user provided information into graph construction and destroy the k-regularity by treating the user provided scribbles as mustlink and must-not-link constraints. However, the user provided constraints are much less than total amount of nodes and this makes the graph structure approximately k-regular.

After modeling the graph structure, the very core problem is to get the edge weight between any pairwise nodes given input data. Most models utilize the $L 2$ distance based Gaussian kernel (See Eq. (1) for example) to define edge weights. However, choosing the optimal Gaussian kernel width parameter is very challenging. Figure 2 illustrates the segmentation sensitivity with respect to the unified kernel width. It can be seen that the kernel width will dramatically influence the final 


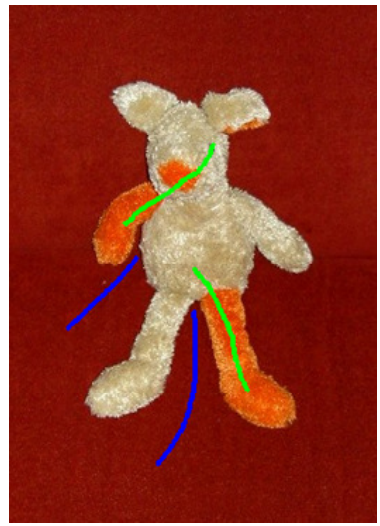

(a)

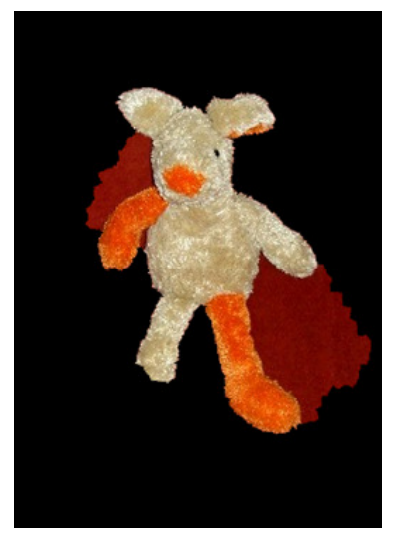

(c)

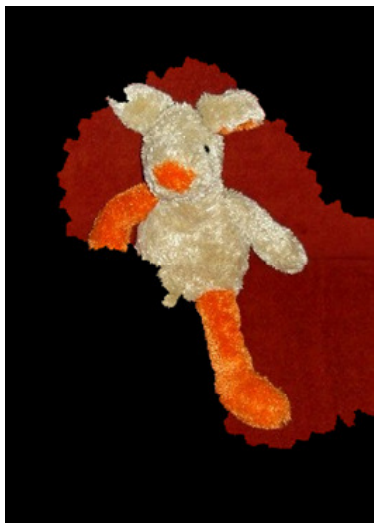

(b)

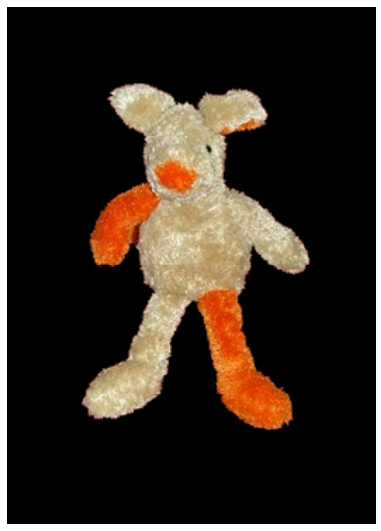

(d)
Fig. 2 Sensitivity of kernel width. (a) Input image with labels. (b) Segmentation result with $\sigma=0.1$. (c) Segmentation result with $\sigma=5$. (d) Segmentation result with $\sigma=10$.

segmentation result and finding the optimal $\sigma$ is usually very hard and time consuming. So in this work, we propose a locally adaptive kernel parameter based edge weight formation strategy, which can be defined as follows:

$$
w_{i j}=\mathrm{e}^{-\frac{d^{2}(i, j)}{\sigma_{i j}}}
$$

where $d(i, j)$ denotes the $L 2$ distance between superpixel region $i$ and $j$ and is defined as

$$
d(i, j)=\left\|c_{i}-c_{j}\right\|^{2}
$$

$c_{i}$ and $c_{j}$ denote the mean of region $i$ and $j$ respectively in $L a b$ color space.

It can also be seen from Eq. (5) that we adopt a content adaptive kernel width instead of a unified one. The reason for this adaption is straightforward: a good choice of $\sigma$ should pull intraclass objects together and push extra-class objects apart simultaneously. Different images have different feature representations and using a globally unified $\sigma$ will not achieve this goal in most time. So we define our local content adaptive kernel width as

$$
\sigma_{i j}=\underset{j \in \mathcal{N}(i)}{\Im}\left(c_{i j}\right)
$$

here $\Im$ denotes the median operation. $\mathcal{N}(i)$ denotes neighboring nodes of superpixel $i$ (all the nodes that have connections with node $i$ ).

Our constructed graph takes spatial relationship, user provided information, and image content into account. It can exploit the intrinsic structure of input data more properly. Algorithm 1 shows our labels driven and locally adaptive graph construction.

Algorithm 1: Labels driven and locally adaptive graph construction

Input: Input image and corresponding superpixles 1: Construct the k-regular graph by extended neighboring relationships.

2: Modify the graph edge connections with mustlink and must-not-link constraints.

3: Compute the distance matrix using $L 2$ distance (Eq. (6)).

4: Form the affinity matrix by Eq. (5) using locally adaptive kernel width.

Output: Affinity matrix.

\subsubsection{Three-stage interactive segmentation}

In this section, we present the details of our threestage strategy for interactive image segmentation with foreground labels and background labels.

Learning with foreground labels. We use the user labeled foreground seeds as queries and other nodes as unlabeled data. By this setting, we get the indicator vector $\boldsymbol{y}$. The ranking scores are learned using Eq. (4). These ranking scores form an $N$ dimensional vector, in which $N$ stands for the number of superpixels (also is the total number of nodes of the graph). Every element in this vector gives the similarity of corresponding node to the foreground queries. Final foreground labels based ranking scores are defined as

$$
R S_{\mathrm{f}}(i)=\bar{f}^{*}(i)
$$

where $i$ is the superpixel index and $\bar{f}^{*}$ is the normalized $f^{*}$ (in the range of $[0,1]$ ).

Learning with background labels. In this stage, we form the indicator vector $\boldsymbol{y}$ by treating the user labeled background seeds as background queries. Then the ranking scores are computed according to Eq. (4) and are normalized into $[0,1]$. 
Final background labels based ranking scores are defined as

$$
R S_{\mathrm{b}}(i)=1-\bar{f}^{*}(i)
$$

where $i$ and $\bar{f}^{*}$ are defined in the same way as in Eq. (8). Note that $\bar{f}^{*}$ are the ranking scores according to background queries, so we subtract them from one to get the corresponding foreground based scores.

Integration. When we get the foreground and background ranking scores, the next stage is to integrate them. In this work, we adopt a very simple strategy defined as

$$
R S_{\mathrm{f}}(i)=\mathcal{M}\left(R S_{\mathrm{f}}(i) * R S_{\mathrm{b}}(i)\right)
$$

where $* *$ stands for pixel-wise product, $R S_{\mathrm{f}}$ and $R S_{\mathrm{b}}$ are defined in Eq. (8) and Eq. (9) respectively, $\mathcal{M}$ denotes mean based thresholding operation defined by

$$
\mathcal{M}\left(f_{i}\right)= \begin{cases}1, & f_{i} \geqslant \mu \\ 0, & f_{i}<\mu\end{cases}
$$

here $\mu$ is the mean value of $\left\{f_{1}, f_{2}, \cdots, f_{N}\right\}$.

Figure 3 shows an example of our three-stage interactive image segmentation algorithm. The detailed procedure can be found in Algorithm 2.

\begin{tabular}{l}
$\begin{array}{l}\text { Algorithm 2: } \\
\text { segmentation }\end{array}$ \\
\hline
\end{tabular}

Input: Input image and user scribbles

1: Construct the graph as stated in Section 3.2.1.

2: Form the foreground and background indicator vectors respectively according to user scribbles.

3: Get the ranking scores by Eq. (8) and Eq. using corredponding indicator $\boldsymbol{y}$.

4: Integrate the ranking scores and get the final segments using Eq. (10).

Output: Final segments.

\section{Experiments and analysis}

In order to show the advantages over previous algorithms, we conduct qualitative and quantitative evaluation on the GrabCut dataset [11] and some real natural images. Firstly, we will analyze the segmentation results with different user scribbles, i.e., sensitivity of user scribbles. Then, we show the flexibility of our framework by extending it to multilabel segmentation and single-line cutout problems. Thirdly, we show the segmentation comparisons

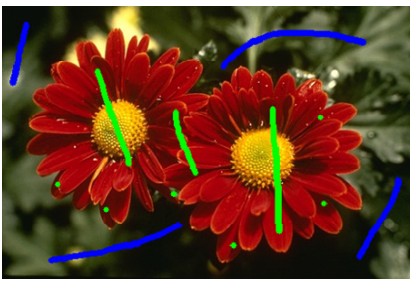

(a)

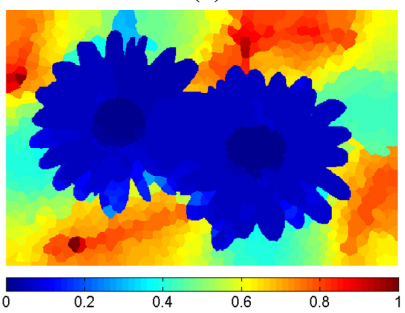

(c)

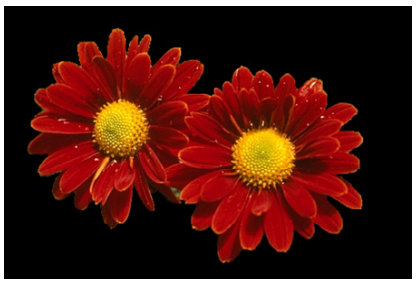

(b)

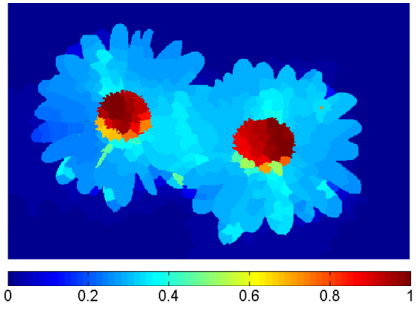

(d)
Fig. 3 Three-stage interactive segmentation. (a) Input image with labels. (b) Segmentation result. (c) Learning with background labels. (d) Learning with foreground labels.

of applying our method and other four methods: RWR [19], GCPP [29], NHL [30], and CAC [17] on some representative images. Finally, we report the running time of these models.

The experimental results of RWR [19], NHL [30], and CAC [17] are all generated by directly using the implementation from the authors. The segmentations of GCPP [29] are generated using our implementation and it is a more recent graph-cut based segmentation framework with post processing output of BJ [10] to remove disconnected foreground islands. To make the comparison more fair and reliable, we keep all the default parameters unchanged and use the same user scribbles. The number of superpixels is set to be $N=500$ in all the experiments. There is one parameter left in our model: the balance weight $\alpha$ in Eq. (4). It balances the smooth and fitting constraints in the regularization. We set $\alpha=0.99$ for all the experiments to put more emphasis on the label consistency like previous graph-based semisupervised learning models usually did. We use green scribbles and blue scribbles to indicate the foreground and background regions respectively in all our experiments except in multi-label segmentation where we use different labels.

\subsection{Comparison of scribble sensitivity}

Through extensive experiments we find that the user scribbles play a very important role in the interactive image segmentation models, i.e., the 
locations and quantity of seeds will drastically affect the segmentation results. So a good interactive segmentation model should be insensitive to the locations and quantity of user scribbles. We demonstrate the user scribble insensitivity of our method in Fig. 4. We use less scribbles in bottom row and the scribbles are also placed in different locations in Fig. 4(a). The corresponding segmentation results of RWR [19], GCPP [29], NHL [30], and CAC [17] are shown in Figs. 4(b)-4(e) respectively. Segmentation results of our method are shown in Fig. 4(f). It can be seen that our method can get almost unchanged best segmentation results given user scribbles of different locations and quantities. Thus our method can generate more stable and satisfying segmentation results that are not sensitive to the locations and quantity of user scribbles.

\subsection{Multi-label segmentation and single-line cutout}

Because we integrate the user scribbles into graph construction and also take spatial relationships into account, our proposed model can be easily extended to the multi-label and single-label segmentation problems in a straightforward manner as illustrated in Fig. 5 and Fig. 6 respectively.

For multi-label segmentation, we use corresponding colors to mask different regions according to the user provided different labels. Take top row in Fig. 5 for example, the left dog and right dog are segmented as red and green regions respectively according to the labels while the rest is treated as background and is labeled as blue just as the label indicated. It can be seen that our method

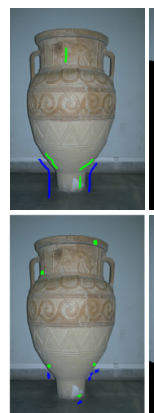

(a)

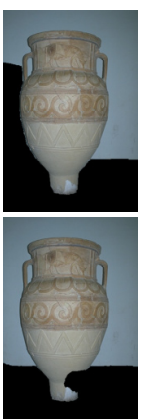

(b)

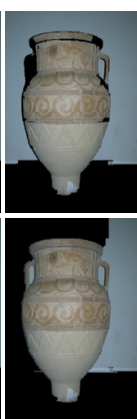

(c)

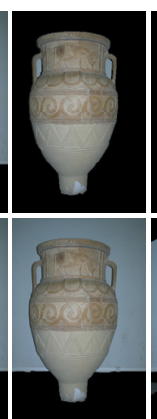

(d) (f)

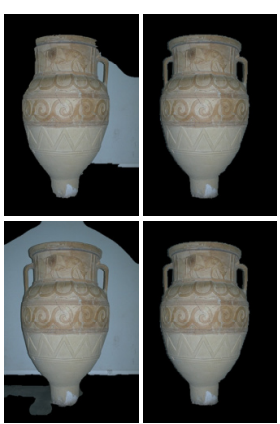

Fig. 4 User scribble sensitivity comparison. (a) Input images with different user scribbles. (b) Results by RWR [19]. (c) Results by GCPP [29]. (d) Results by NHL [30]. (e) Results by CAC [17]. (f) Our results.
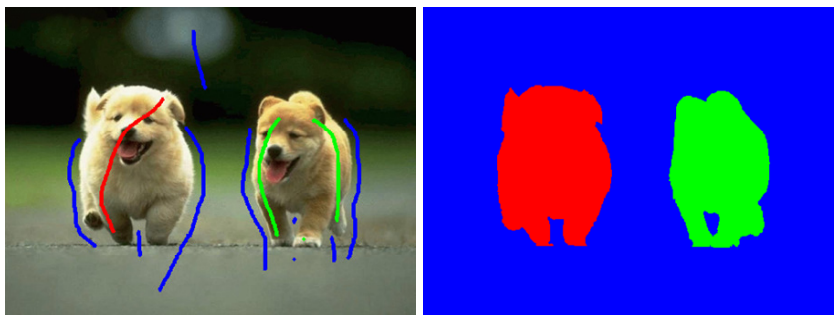

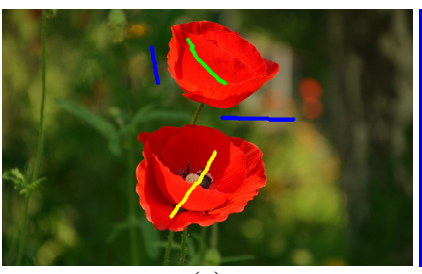

(a)

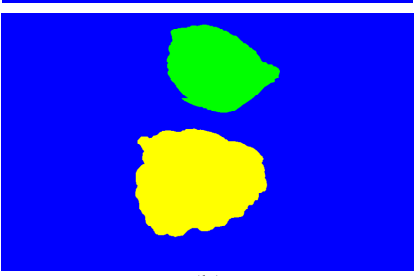

(b)
Fig. 5 Multi-label segmentation. (a) Input images with multilabels. (b) Corresponding segmentation results.
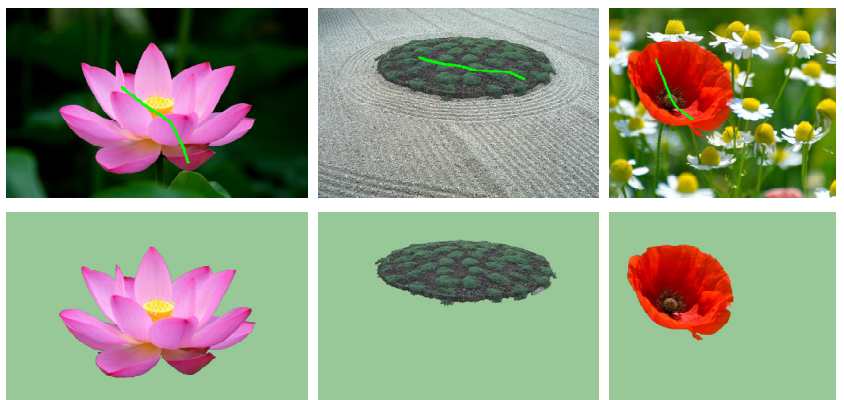

Fig. 6 Single-line cutout. Top row: Input images with singleline label (only foreground labels). Bottom row: Corresponding segmentation results.

can generate highly accurate segmentations, even in the presence of multiple objects with similar color.

For single-label segmentation problem, which we refer as single-line cutout, it just uses the foreground label to segment out the desired object. As shown in Fig. 6, it can get satisfying segmentation results using only single-line interaction. This will definitely make the segmentation problem more convenient and interesting.

\subsection{Qualitative and quantitative comparison}

In Fig. 7, the segmentations are produced by five algorithms including RWR [19], GCPP [29], NHL [30], CAC [17], and ours. It shows the qualitative and quantitative comparison of these five different algorithms.

For qualitative comparison, we use the same user scribbles and corresponding optimal parameters to generate the segmentation results. Then for each segmention, we form its boundary map and integrate 

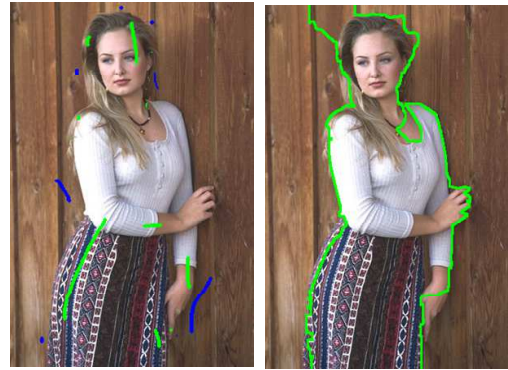

$\beta_{0}=0.8783$
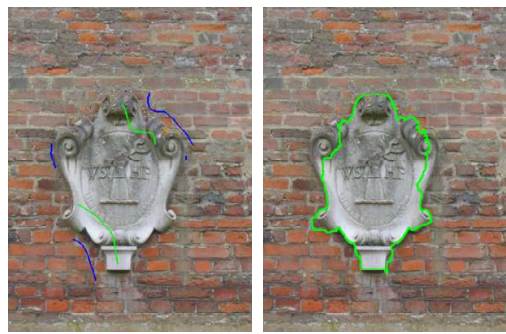

$\beta_{\mathrm{o}}=0.8617$
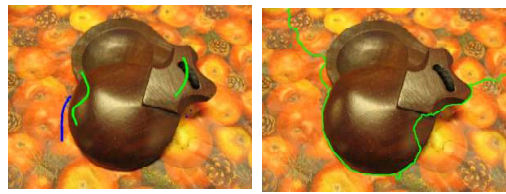

$\beta_{\mathrm{o}}=0.6174$
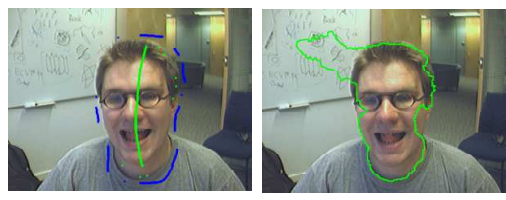

$\beta=0.7616$
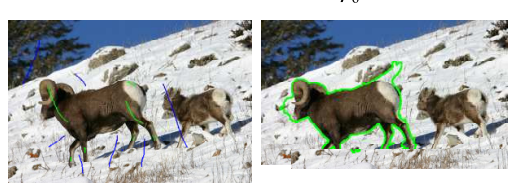

$\beta_{\mathrm{o}}=0.8695$

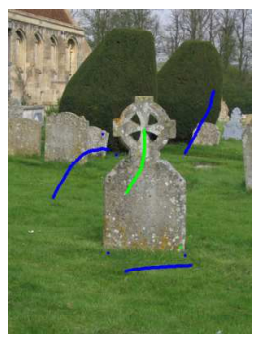

(a)

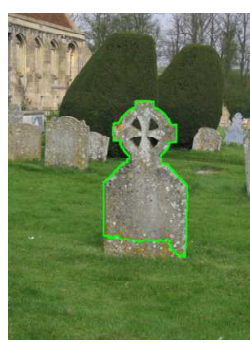

$\beta_{\mathrm{o}}=0.8554$

(b)

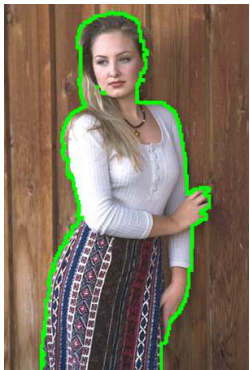

$\beta_{\mathrm{o}}=0.9599$

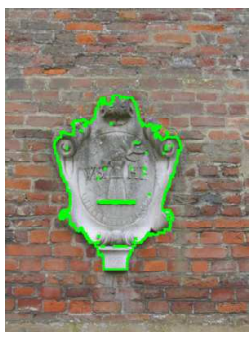

$\beta_{\mathrm{o}}=0.9236$

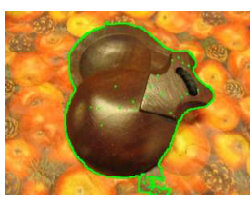

$\beta_{\mathrm{o}}=0.9523$

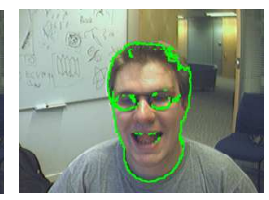

$\beta_{\mathrm{o}}=0.9210$

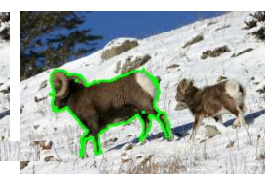

$\beta_{\mathrm{o}}=0.9360$

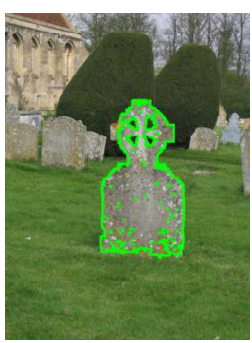

$\beta_{\mathrm{o}}=0.9136$

(c)

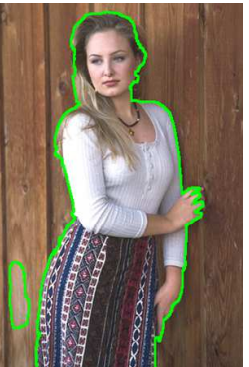

$\beta_{\mathrm{o}}=0.9393$

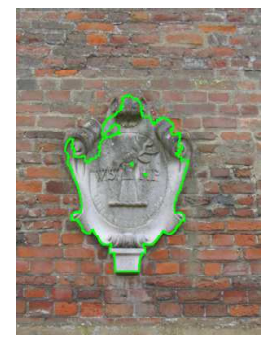

$\beta_{\mathrm{o}}=0.8417$

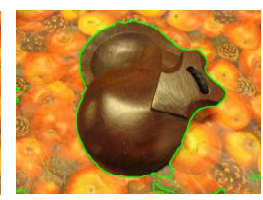

$\beta_{\mathrm{o}}=0.9585$

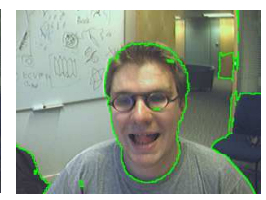

$\beta_{\mathrm{o}}=0.6436$
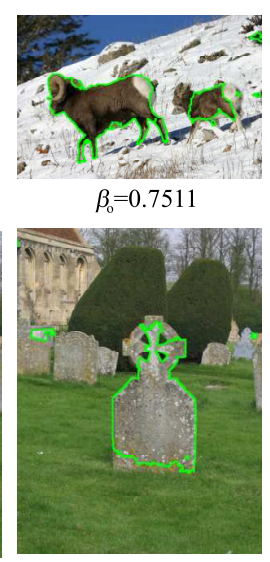

$\beta_{\mathrm{o}}=0.8073$

(d)

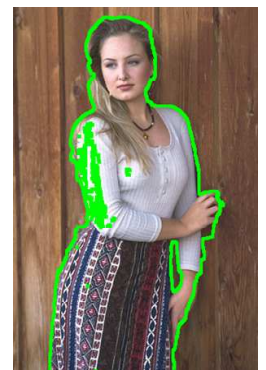

$\beta_{\mathrm{o}}=0.9270$

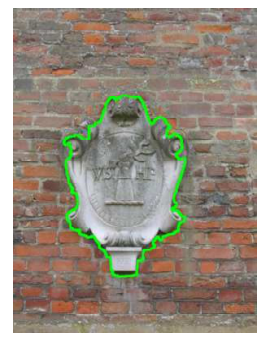

$\beta_{\mathrm{o}}=0.8914$

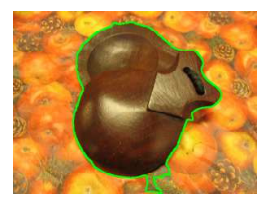

$\beta_{\mathrm{o}}=0.9611$

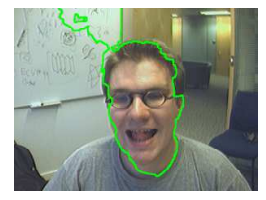

$\beta_{\mathrm{o}}=0.7308$
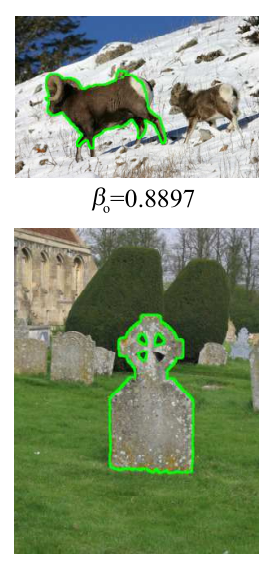

$\beta_{\mathrm{o}}=0.9644$

(e)

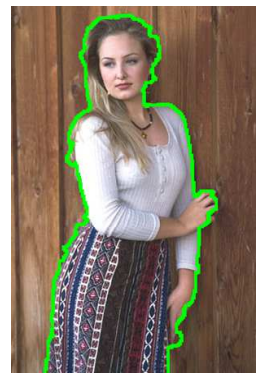

$\beta_{\mathrm{o}}=0.9665$

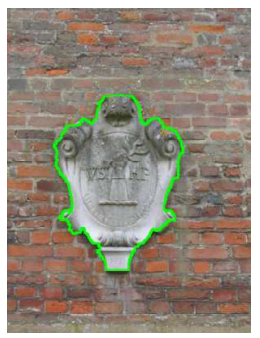

$\beta_{\mathrm{o}}=0.9644$

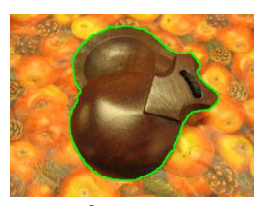

$\beta_{\mathrm{o}}=0.9827$

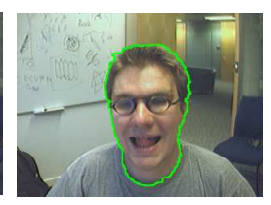

$\beta_{\mathrm{o}}=0.9627$
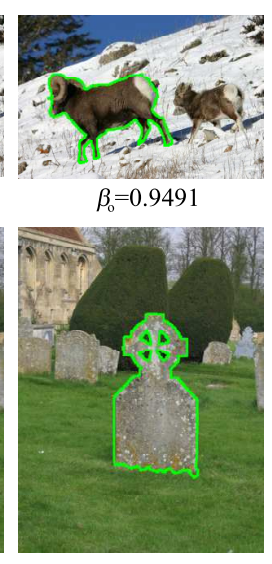

$\beta_{\mathrm{o}}=0.9682$

(f)

Fig. 7 Comparison of our model with other models. (a) Input images with labels. (b) Results by RWR [19]. (c) Results by GCPP [29]. (d) Results by NHL [30]. (e) Results by CAC [17]. (f) Our results.

it into input image to visualize the performance of segmentation. Figures 8 and 9 give the fair comparisons of more complicated images from the GrubCut dataset [11].
For quantitative comparison, we use the normalized overlap $\beta_{\mathrm{o}}$ [31] to measure the similarity between the segmentation result and the preset ground truth quantitatively. It is defined as 

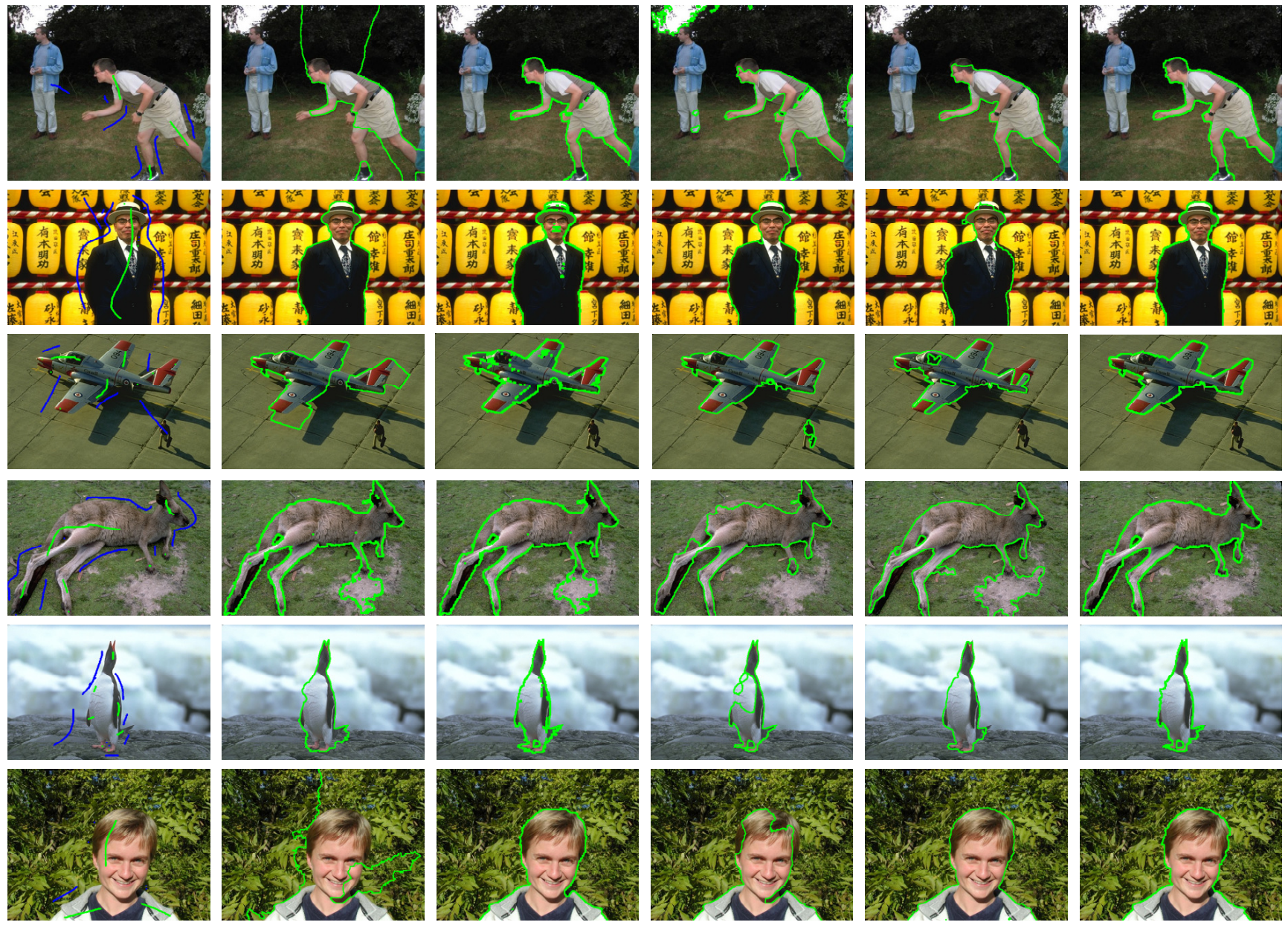

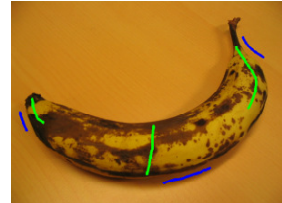

(a)

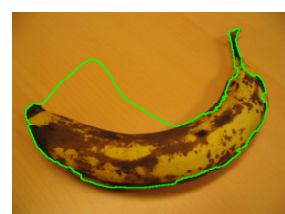

(b)

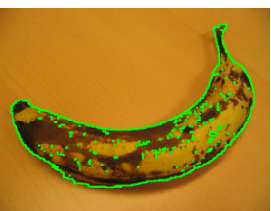

(c)

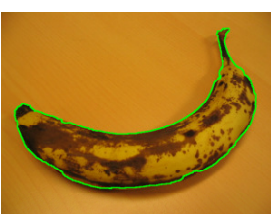

(d)

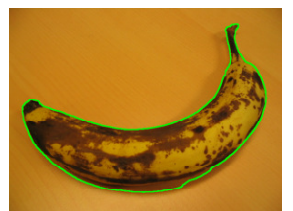

(e)

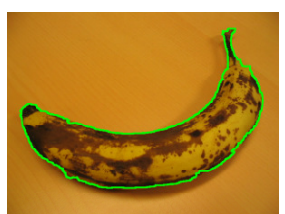

(f)

Fig. 8 Comparison of our model with other models. (a) Input images with labels. (b) Results by RWR [19]. (c) Results by GCPP [29]. (d) Results by NHL [30]. (e) Results by CAC [17]. (f) Our results.

$$
\beta_{\mathrm{o}}=\frac{\left|S_{\mathrm{f}} \cap G_{\mathrm{f}}\right|}{\left|S_{\mathrm{f}} \cup G_{\mathrm{f}}\right|}
$$

where $S_{\mathrm{f}}$ is the assigned foreground pixels of the segmentation result and $G_{\mathrm{f}}$ is that of ground truth. This value is presented below each segmentation result.

As can be seen, RWR [19] and GCPP [29] can generally generate satisfactory segmentation results. However, RWR [19] can only get good segmentation results when there are enough user scribbles to surround the desired object. This requirement makes their method inapplicable because it needs more user scribbles. For GCPP [29], it will produce isolated regions (even dots) in bigger foreground regions as shown in the fourth and last rows of the third column. CAC [17] will segment out background regions when the background and foreground have similar colors. NHL [30] has the problem of producing isolated regions and segmenting out background regions when the corresponding regions have no scribbles. On the other hand, our model consistently outperforms all other models. It has the highest $\beta_{\mathrm{o}}$ value which indicates that the segmentation results are more consistent with ground truth. Meanwhile, the segmentation results of our model have high boundary fitness. 


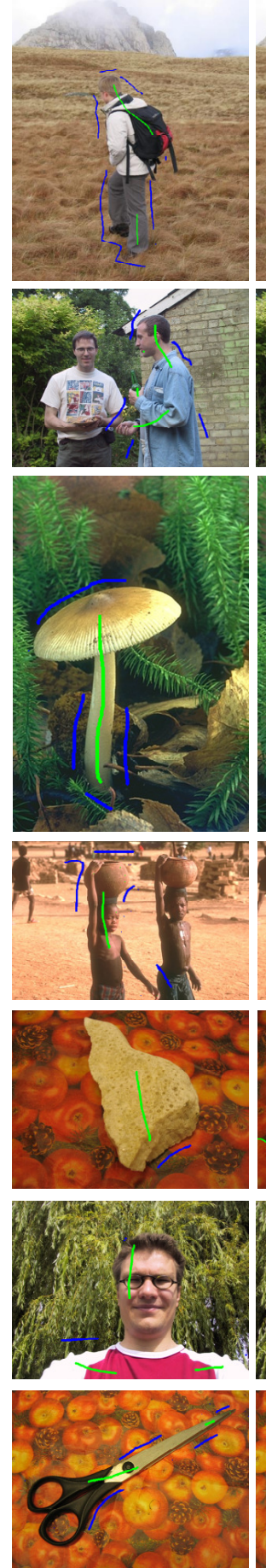

(a)
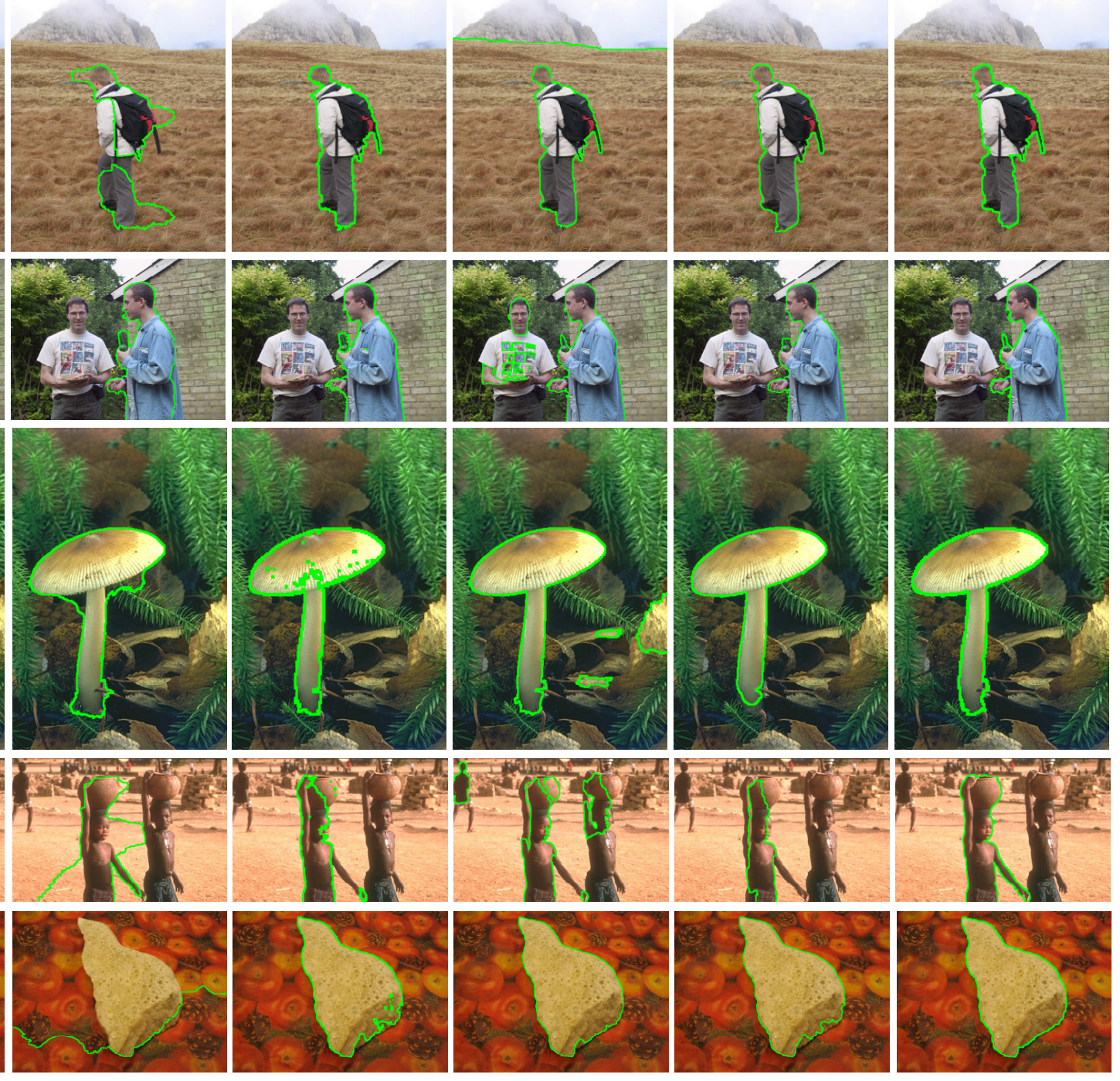

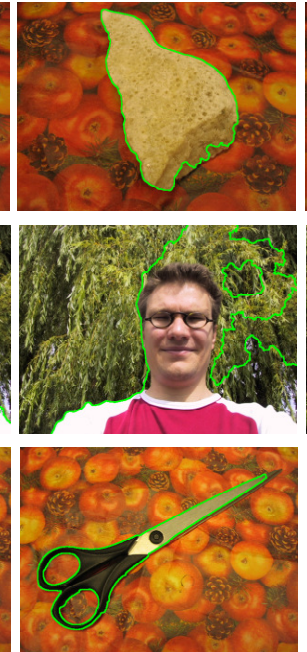

(e)

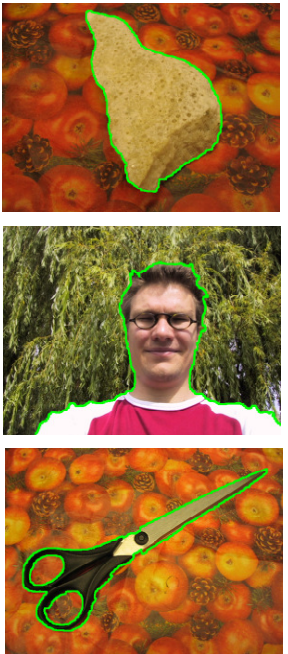

(d)

Fig. 9 Comparison of our model with other models. (a) Input images with labels. (b) Results by RWR [19]. (c) Results by GCPP [29]. (d) Results by NHL [30]. (e) Results by CAC [17]. (f) Our results.

\subsection{Running time}

Another very important factor which will influence the level of segmentation satisfaction dramatically is the computational cost. The processing should be very fast in order to let the users modify the segmentation results in a real-time fashion. We conduct experiments on some representative images and report the mean running time of each model. All 
the experiments are carried out on a PC with an Intel Core i7 $3.2 \mathrm{GHz}$ processor and $16 \mathrm{~GB}$ of RAM. Table 1 illustrates the running time of different models for segmentations on images with size of $640 \times 480$.

We can see from Table 1 that NHL [30] needs the most time; it takes about fifty seconds to process an image. The rest four models including ours need almost the same time to proceed. It's worth mentioning that our unoptimized MATLAB code only needs less than 2 seconds including oversegmentation computation time to segment the input image. The running time of our model can be sharply reduced by standard multi-core methods due to the sparsity of our model in $\mathrm{C}++$ implementation and it can provide real-time performance. This will be one of our next works. We will make all the resources including source code, dataset, and evaluation code publicly available after this paper been published.

\section{Conclusions and further work}

In this paper, we have proposed a novel framework for interactive image segmentation, which generates accurate segmentation results with very fast respond to users' interactions. The core of this technique is a graph-based semi-supervised learning framework to rank similarities of unlabeled data points with respect to the labeled ones by exploiting the global and local consistency of all the data.

To better exploit the intrinsic structure of data, we firstly model the graph as a k-regular graph to take spatial relationships into account. Then we further enhance the graph structure by integrating user provided scribbles and finally model the graph as an approximately k-regular sparse graph. To overcome the instability brought by the sensitivity of hyper-parameter, we propose a content based locally adaptive kernel width parameter to form the graph edge weights. A three-stage strategy is employed to generate the final segmentation

Table 1 Running time of different models

\begin{tabular}{ccc}
\hline Model & Programming language & Time $(\mathrm{s})$ \\
\hline NHL & MATLAB & 48.79 \\
CAC & C ++ & 2.8 \\
RWR & MATLAB & 3.13 \\
GCPP & MATLAB/C++ & 2.1 \\
Ours & MATLAB & $\mathbf{1 . 9 8}$ \\
\hline
\end{tabular}

results. Our framework can also be easily extended to multi-label segmentation and single-line cutout problems. Extensive experiments show that our model consistently outperforms other models both qualitatively and quantitatively. Last but not least, our framework has the least computational cost compared with other four models due to the sparsity of our constructed graph and usage of superpixels.

As future work, we consider two possible directions: multi-features, multi-scale and optimization. We only use color feature for now. There are other features that can be integrated into this framework to better differentiate different regions, such as texture and edge. We employ superpixels as our basic processing unit. The incorrect over-segmentation will affect the final segmentation result. This disadvantage can be overcome effectively by utilizing the multiscale technique. We will further optimize the framework and consider parallelism to speed up the segmentation procedure.

\section{Acknowledgements}

The authors would like to thank the anonymous reviewers for their valued suggestions which helped a lot to improve the manuscript. This work has been supported by NSFC (National Natural Science Foundation of China, No. 61272326), the research grant of University of Macau (No. MYRG202(Y1L4)-FST11-WEH), and the research grant of University of Macau (No. MYRG2014-00139-FST).

Open Access This article is distributed under the terms of the Creative Commons Attribution License which permits any use, distribution, and reproduction in any medium, provided the original author(s) and the source are credited.

\section{References}

[1] Hu, S.-M.; Chen, T.; Xu, K.; Cheng, M.-M.; Martin, R. R. Internet visual media processing: A survey with graphics and vision applications. The Visual Computer Vol. 29, No. 5, 393-405, 2013.

[2] Comaniciu, D.; Meer, P. Mean shift: A robust approach toward feature space analysis. IEEE Transactions on Pattern Analysis and Machine Intelligence Vol. 24, No. 5, 603-619, 2002.

[3] Xiao, C.; Liu, M. Efficient mean-shift clustering using 
Gaussian KD-tree. Computer Graphics Forum Vol. 29, No. 7, 2065-2073, 2010.

[4] Vese, L. A.; Chan, T. F. A multiphase level set framework for image segmentation using the Mumford and Shah model. International Journal of Computer Vision Vol. 50, No. 3, 271-293, 2002.

[5] Li, C.; Xu, C.; Gui, C.; Fox, M. D. Distance regularized level set evolution and its application to image segmentation. IEEE Transactions on Image Processing Vol. 19, No. 12, 3243-3254, 2010.

[6] Liu, Y.; Yu, Y. Interactive image segmentation based on level sets of probabilities. IEEE Transactions on Visualization and Computer Graphics Vol. 18, No. 2, 202-213, 2012.

[7] Shi, J.; Malik, J. Normalized cuts and image segmentation. IEEE Transactions on Pattern Analysis and Machine Intelligence Vol. 22, No. 8, 888-905, 2000 .

[8] Huang, H.; Zhang, L.; Zhang, H.-C. RepSnapping: Efficient image cutout for repeated scene elements. Computer Graphics Forum Vol. 30, No. 7, 2059-2066, 2011.

[9] Eigen, D.; Fergus, R. Nonparametric image parsing using adaptive neighbor sets. In: Proceedings of 2012 IEEE Conference on Computer Vision and Pattern Recognition, 2799-2806, 2012.

[10] Boykov, Y. Y.; Jolly, M.-P. Interactive graph cuts for optimal boundary \& region segmentation of objects in N-D images. In: Proceedings of the Eighth IEEE International Conference on Computer Vision, Vol. 1, 105-112, 2001.

[11] Rother, C.; Kolmogorov, V.; Blake, A. "GrabCut": Interactive foreground extraction using iterated graph cuts. ACM Transactions on Graphics Vol. 23, No. 3, 309-314, 2004

[12] Li, Y.; Sun, J.; Tang, C.-K.; Shum, H.-Y. Lazy snapping. ACM Transactions on Graphics Vol. 23, No. 3, 303-308, 2004.

[13] Price, B. L.; Morse, B.; Cohen, S. Geodesic graph cut for interactive image segmentation. In: Proceedings of the 2010 IEEE Conference on Computer Vision and Pattern Recognition, 3161-3168, 2010.

[14] Gulshan, V.; Rother, C.; Criminisi, A.; Blake, A.; Zisserman, A. Geodesic star convexity for interactive image segmentation. In: Proceedings of the 2010 IEEE Conference on Computer Vision and Pattern Recognition, 3129-3136, 2010.

[15] Mortensen, E. N.; Barrett, W. A. Intelligent scissors for image composition. In: Proceedings of the 22nd Annual Conference on Computer Graphics and Interactive Techniques, 191-198, 1995.

[16] Sundaramoorthi, G.; Yezzi, A.; Mennucci, A. C. Coarse-to-fine segmentation and tracking using Sobolev active contours. IEEE Transactions on Pattern Analysis and Machine Intelligence Vol. 30, No. 5, 851-864, 2008.
[17] Nguyen, T. N. A. N.; Cai, J.; Zhang, J.; Zheng, J. Robust interactive image segmentation using convex active contours. IEEE Transactions on Image Processing Vol. 21, No. 8, 3734-3743, 2012.

[18] Grady, L. Random walks for image segmentation. IEEE Transactions on Pattern Analysis and Machine Intelligence Vol. 28, No. 11, 1768-1783, 2006.

[19] Kim, T. H.; Lee, K. M.; Lee, S. U. Generative image segmentation using random walks with restart. In: Lecture Notes in Computer Science, Vol. 5304. Berlin Heidelberg: Springer, 264-275, 2008.

[20] Yang, W.; Cai, J.; Zheng, J.; Luo, J. Userfriendly interactive image segmentation through unified combinatorial user inputs. IEEE Transactions on Image Processing Vol. 19, No. 9, 2470-2479, 2010.

[21] Adams, R.; Bischof, L. Seeded region growing. IEEE Transactions on Pattern Analysis and Machine Intelligence Vol. 16, No. 6, 641-647, 1994.

[22] Ning, J.; Zhang, L.; Zhang, D.; Wu, C. Interactive image segmentation by maximal similarity based region merging. Pattern Recognition Vol. 43, No. 2, 445-456, 2010.

[23] Noma, A.; Graciano, A. B. V.; Cesar Jr., R. M.; Consularo, L. A.; Bloch, I. Interactive image segmentation by matching attributed relational graphs. Pattern Recognition Vol. 45, No. 3, 1159-1179, 2012.

[24] Greig, D. M.; Porteous, B. T.; Seheult, A. H. Exact maximum a posteriori estimation for binary images. Journal of the Royal Statistical Society. Series B (Methodological) Vol. 51, No. 2, 271-279, 1989.

[25] Zhou, D.; Bousquet, O.; Lal, T. N.; Weston, J.; Schölkopf, B. Learning with local and global consistency. In: Proceedings of Advances in Neural Information Processing Systems, 321-328, 2003.

[26] Zhou, D.; Weston, J.; Gretton, A.; Bousquest, O.; Schölkopf, B. Ranking on data manifolds. In: Proceedings of Advances in Neural Information Processing Systems, 2004. Available at http://papers.nips.cc/paper/2447-ranking-ondata-manifolds.pdf.

[27] Shen, J.; Du, Y.; Wang, W.; Li, X. Lazy random walks for superpixel segmentation. IEEE Transactions on Image Processing Vol. 23, No. 4, 1451-1462, 2014.

[28] Achanta, R.; Shaji, A.; Smith, K.; Lucchi, A.; Fua, P.; Susstrunk, S. SLIC superpixels compared to stateof-the-art superpixel methods. IEEE Transactions on Pattern Analysis and Machine Intelligence Vol. 34, No. 11, 2274-2282, 2012.

[29] Liu, J. Y.; Sun, J.; Shum, H. Y. Paint selection. In: Proceedings of ACM SIGGRAPH 2009 papers, Article No. 69, 2009.

[30] Kim, T. H.; Lee, K. M.; Lee, S. U. Nonparametric higher-order learning for interactive segmentation. In: Proceedings of 2010 IEEE Conference on Computer Vision and Pattern Recognition, 3201-3208, 2010. 
[31] Sinop, A. K.; Grady, L. A seeded image segmentation framework unifying graph cuts and randomwalker which yields a new algorithm. In: Proceedings of IEEE 11th International Conference on Computer Vision, 18, 2007.

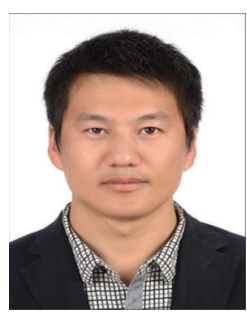

Hong Li is currently a third-year Ph.D. candidate at Macau University (Macao, China), Computer Graphics Laboratory. His researches focus on image processing, semi-supervised learning, computer vision, and so on.

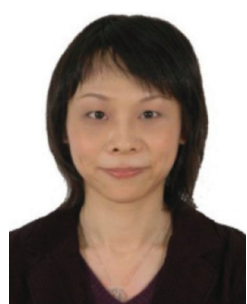

Wen Wu received her Ph.D. degree in computer science and engineering from The Chinese University of Hong Kong. She is currently an assistant professor in Department of Computer and Information Science at University of Macau. Her research interests include medical simulation, virtual reality, and physically-based animation.

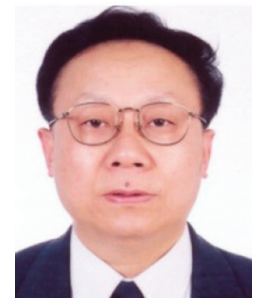

Enhua Wu is a professor of the State Key Laboratory of Computer Science at the Chinese Academy of Sciences Institute of Software and the University of Macau. His interests include VR, realistic-image synthesis, and data visualization. He received his Ph.D. in computer science from University of

Manchester.

Other papers from this open access journal are available free of charge from http://www.springer.com/journal/41095. To submit a manuscript, please go to https://www. editorialmanager.com/cvmj. 